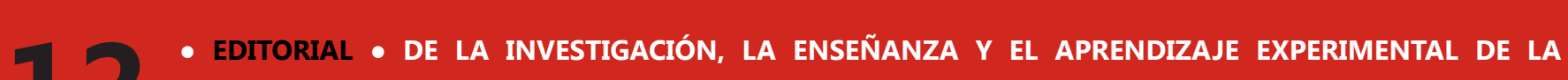
ARQUITECTURA / FROM RESEARCH, TEACHING AND THE EXPERIMENTAL LEARNING OF ARCHITECURE. Amadeo Ramos CarranZa • ENTRE LínEAS • LA ARQUITECTURA COMO MODO DE ENTENDER EL MUNDO. NOTAS DE UN PROFESOR VETERANO / ARCHITECTURE AS A WAY TO UNDERSTAND THE WORLD. NOTES FROM A VETERAN PROFESSOR. Antonio González-Capitel - ARTículos • LA PALABRA DIBUJADA. ANTONIO FERNÁNDEZALBA, PRIMER Y ÚLTIMO MAESTRO / THE SKETCHED WORD. ANTONIO FERNÁNDEZ-ALBA, THE FIRST AND LAST MASTER. Juan Luis Trillo de Leyva • CARVAJAL Y LA VOLUNTAD DE SER ARQUITECTO: LA CONSTRUCCIÓN DEL PROYECTO Y LA BELLEZA EFICAZ / CARVAJAL AND THE WILL TO BE ARCHITECT: THE CONSTRUCTION OF THE PROJECT AND EFFECTIVE BEAUTY. Carlos Labarta Aizpún; Jorge Tárrago Mingo • CIUDAD BLANCA EN BAHÍA DE ALCUDIA. UNA OBRA CON SENTIDO PEDAGóGICO DEL PROFESOR FRANCISCO JAVIER SÁENZ DE OÍZA. 1961-63 / CIUDAD BLANCA IN ALCUDIA BAY. AN EDUCATIONAL WORK BY PROFESSOR FRANCISCO JAVIER SÁENZ DE OíZA. 1961-63. Rosa María Añón Abajas; Salud María Torres Dorado • SEVILLA Y EL SEVILLA 1(1972-2015) / SEVILLE AND THE SEVILLE 1 (1972-2015). Valentín Trillo Martínez • DE LA PROFESIÓN A LA DOCENCIA: LOS VIAJES A INGLATERRA DE MANUEL TRILLO Y LAS VIVIENDAS EN LA MOTILLA / FROM PROFESSION TO TEACHING: MANUEL TRILLO AND HIS TRIPS TO ENGLAND AND THE COLLECTIVE HOUSING IN LA MOTILLA. Amadeo Ramos Carranza; José Altés Bustelo • LA CONDICIÓN TERRITORIAL DE LO URBANO. EN TORNO A LA TRAYECTORIA DOCENTE DE PABLO ARIAS / THE CITY WITHIN THE FRAME OF TERRITORY. ABOUT THE ACADEMIC CAREER OF PABLO ARIAS. Victoriano Sainz Gutiérrez • RESEÑAS BIBLIOGRÁFICAS • MANUEL TRILLO DE LEYVA: LA EXPOSICIÓN IBEROAMERICANA: LA TRANSFORMACIÓN URBANA DE SEVILLA. Alfonso del Pozo y Barajas • MANUEL TRILLO DE LEYVA: CONSTRUYENDO LONDRES; DIBUJANDO EUROPA. Tomás Curbelo Ranero; Manuel Ramos Guerra

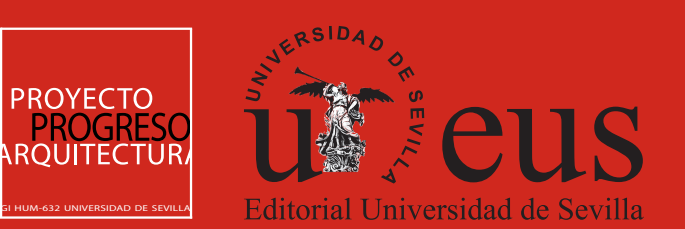

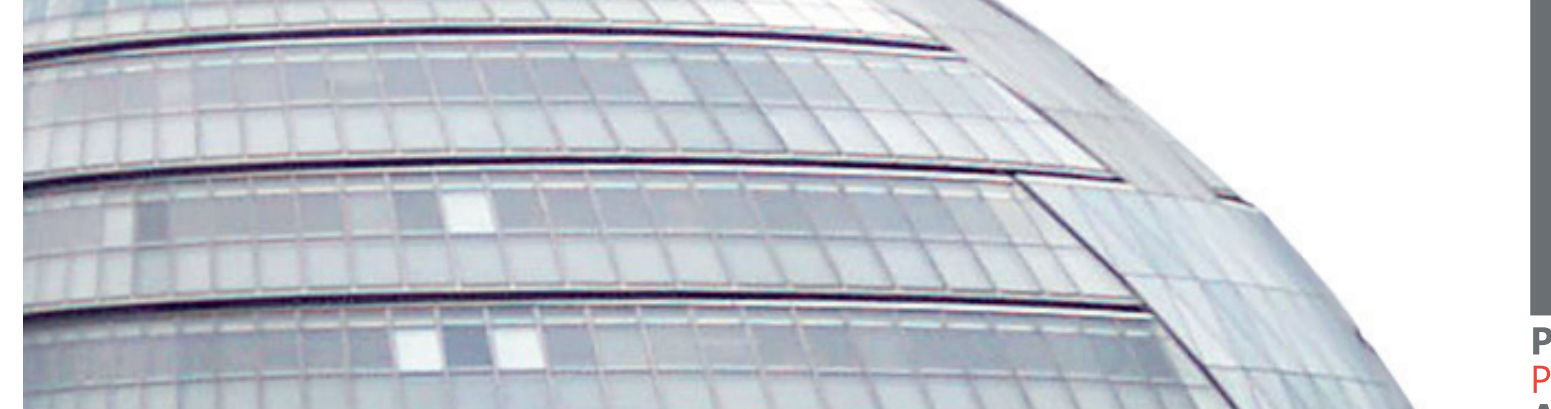

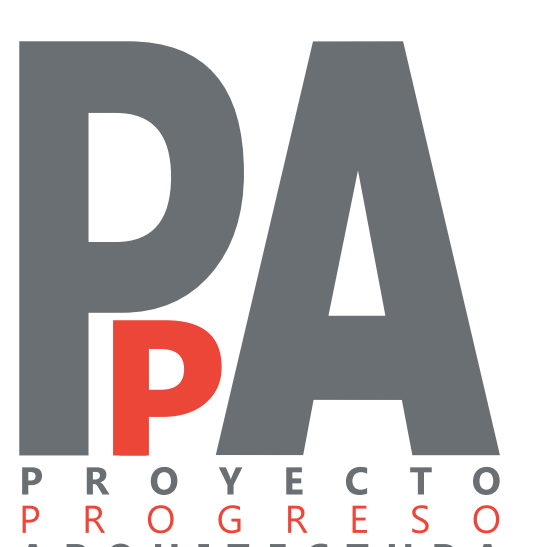

A R Q U I T E C T U R A
ARQUITECTOS Y PROFESORES 
DIRECCIÓN

Escuela Técnica Superior de

Arquitectura. Universidad de Sevilla

SECRETARIA

Dr. Rosa María Añón Abajas. Escuela Técnica Superior de Arquitectura Universidad de Sevilla

Dr. Rosa María Añón Abajas. Escuela Técnica Superior de Arquitectura Universidad de Sevilla. España. Miguel Ángel de la Cova Morillo-Velarde. Escrantectura. Universidad de Sevilla. España

Arquitectura. Universidad de Sevilla. España. Juan José López de la Cruzz. Escuela Tecnica Superior de Arqu
Universidad de Sevilla. España. Germán López Mena. Escuela

Universidad de Sevilla. Españá. Arquitectura. Universidad de Sevilla. Espanan

nica Superior de Arquitectura. Dr. Alfonsad de Sevilla. España .

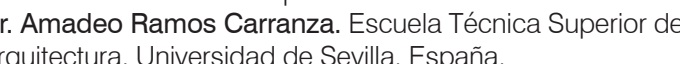

COMITÉ CIENTÍFICO

Dr. Gonzalo Díaz Recaséns. Catedrático Proyectos Arquitectónicos Escuela Técnica Superior de Arquitectura. Universidad de Sevilla. Espan Escusela Mécnica Superior de Arquitectura Universidad Politécnicán de Madrid. España.

Dr. Víctor Pérez Escolano. Catedrático Historia, Teoría y Composición Arquitectónicas. Escuela Técnica Superior de Arquitectura. Universidad r. Jorge Torres $\mathrm{C}$

Técnica Superior de Arquitectura. Universididad de Valenciai España Dr. Armando Dal'Fabbro. Profosessore Associato. Dipartimento di

progettazione architettonica, Facoltà di Architettura, Universitat Instituto

Dr. Mario Coyula Cowley. Profesor de Mérito en la Facultad de
Arcuitectura, del Instituto Superior Politécnico José Antonio Echever Cuba.

Dr. Anne-Marie Chatelêt. Professeur Titulaire. Histoire et Cultures Architecturales. École Nationale Supérieure d'Architecture de Versailles.
Francia CONSEJO ASESOR

Alberto Altes Arlandis. Escola d'Arquitectura del Vallès. Universitat Politécnica de Catalunya. Espana

Dr. José Altés Bustelo. Escuela Técnica Superior de Arquitectura

Dr. José de Coca Leicher. Escuela de Arquitectura y Geodesi

Universidad de Alcala de Henares. España

Dr. Jaume J. Ferrer Fores. Escola Tècnica Superior d'Arquitectura de

Barcelona. Universitat Politècnica de Catalunya. España

Atlántico. Colombia.

Carmen Peña de Urqú́ architect en RSH-P. Londres. Reino Unido.

ISSN-ed. impresa: 2171-6897

ISSN-ed. electrónica: 2173-1616

DOl: http:///dx.doi.org/10.12795/ppa

DEPÓSITO LEGAL: SE-2773-2010
PERIOCIDAD DE LA REVISTA: MAYOY

IMPRIME: TECNOGRAPHIC S.L.
EDITA

AQUETA DE LA PORTADA

Miguel Ángel de la Cova Morillo-Velarde.

DISEÑO DE LA PORTADA

DTOGRAF́́A DE LA PORTADA

, (Londres, junio 2003).

DISEÑO GRÁFICO Y DE LA MAQUETACIÓN

inodríguez

COLABORACIÓN EN EL DISEÑO DE LA PORTADA Y MAQUETACIÓN

Alvaro Borrego Plata.

E.T.S. de Arquitectura. Avda Reina Mercedes, no 241012 -Sevilla.

Amadeo Ramos Carranza, Dpto. Proyectos Arquitectónicos.

EDICIÓN ON-LINE

Portal intormatico https://ojs. publius.us.es/ojs/index.php/ppa/index

Portalinformático G.I.IUM-632http://muw.proyectoprogresoarquitectura.com

hortta://nww.ermático Editorial.us.es/

(c) EDITORIAL UNIIERSIDAD DE SEVILLA.

Calle Porvenir, 27. 41013 SEVILLA. Tfs. $954487447 / 95448745$

๑ TEXTOS: SUS AUTORES.

( $)$ IMAGENES: SUS AUTORES Y/O INSTITUCIONES.

SUSCRIPCIONES, ADQUISICIONES Y CANJE

EVISTa PROYECTO, PROGRESO, ARQUITECTURA

Caltorial Ponivenirsididad de Sevilla. 41013 SEVILLA. Tfs. 954487447 / 95448745

Reservados todos los derechos. Ni la totalidad ni parte de esta revista puede reproducirse otransmititise por ningun procedimiento electrónico o mecánico,
incluyendo fotocopia grabación magnética o cualquier almacenamiento de información y sistema de recuperación, sin permiso escrito del Secretariado de Publicaciones de la Universidad de Sevilla

tores en los artículos firmados son responsabilidad exclusiva de los mismos.

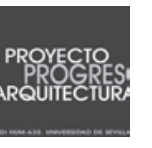

INICIATIVA DEL GRUPO DE INVESTIGACION HUM-632 ittp.//mmw proyectoprogresoarquitecturacon

COLABORA EL DEPAATAMENTO DE PROYECTOS ARQUITECTÓNIICOS hittp://wnw departamento us es/dpetses

\section{revista PROYECTO, PROGRESO, ARQUITECTURA}

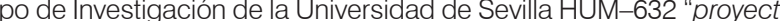
progreso, arqu lecthra t tiene por objetivo compartir y debatir sobre investigación en arquitectura. Es una publicación cientifica con (a) sistema doble ciego-siguiendo los protocolos habituales para publicaciones cientfficas seriadas. Los fitulos, resúmenes y palabras "proyecto, progreso, arquitectura" presenta una estructura clara, sencilla y flexible. Trata todos los temas relacionados con la teoria
la práctica del proyecto arquitectónico. Las distintas "temáticas abiertas" que componen nuestral linea editorial, son las fuentes para de arquitectura. Dur Journal, "proyecto, progreso, arquitectura", founded in 2010, is an inititative of the Research Group HUM-632 of the University of Sevilie and its objective is the sharing and debating of research within architecture. This six-monthly scientific publication, in paper and digital format, publishes original works that have not been previously pubilshed in other journals. Tiene article silection process summaries and key words of articles are also published in English. 作 diverse investigations.

(the accomplishment of

\section{SISTEMA DE ARBITRAJE}

VALUACION EXTERNA POR PARES Y ANÓNIM

En lansejo Eticorial de la revista, una vez comprobado que el artículo cumple con las normas relativas a estilo y contenido indicadas y critica de arquitectura, según el modelo doble ciego.

政 evaluación por correo electrónico, en la dirección que éstos hayan utilizado para enviar el artículo. El director comunicará al autor importantes; no aconsejable para su publicación), así como las observaciones y comentarios de los revisores.
intion Si el manuscrito ha sido aceptado con modificaciones, los autores deberán reenviar una nueva versión del artículo, atendiendo a las demandas y sugerencias de los evaluadores externos. Si lo desean, los autores pueden aportar también una carta al Consejo Editorial en la que indicarán el contenido de las modificaciones del artículo. Los articulos con correcciones import
Consejo Asesor y/o Cientffico para verfificar la validez de las modificaciones efectuadas por el autor.

EXTERNAL ANONYMOUS PEER REVIEW

instructions for authors, the article will be sent to two anonymous experts, within the specific field of architectural investigation and critique, for a double blind review

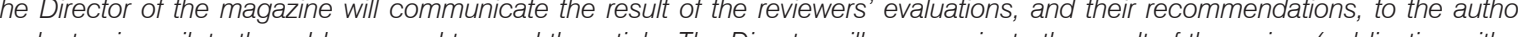
by electronic mall, to the address used to send the article. The Difeclor wil communicate he resull or he review (publicalion withou observations and comments of the reviewers, to the main author. If the manuscript has been accepted with modifications, the authors will have to resubmit a new version of the article, addressing the

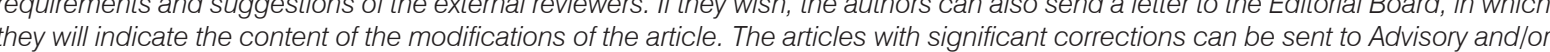
Scientific Board for verification of the valididity of the modifications made by the author

\section{INSTRUCCIONES A AUTORES PARA LA REMISIÓN DE ARTÍCULOS}

NORMAS DE PUBLICACIÓN

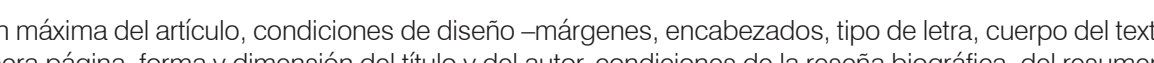
de las palabras claves, de las citas, de las imágenenes -numeración en texto, en pié de imágesenes, calidad de la imagen y autoría procedencia-y de la bibliografía en http://www.proyectoprogresoarquitectura.com

PUBLICATION STANDARDS

instructions to authors: maximum length of the article, design conditions (margins, headings, font, body of the text and quotations, key words, quotations, images (text numeration, image captions, image quality and authorship or origin) and of the bibliography in http " unw.proyectoprogresoarquitectura.com 


\section{SERVICIOS DE INFORMACIÓN}

La Editorial Universidad de Sevilla cumple los criterios establecidos por la Comisión Nacional Evaluadora de la Actividad hivestigadora para que lo publicado por el milsmo sea reconocido como de impacto "Ministerio de Clencia e Innovación. a Editorial Universidad de Sevilla forma parte de la UN E (Unión de Editoriales Universitarias Españolas) ajustáncose a s sistem de control de calidad que garantiza el prestigio e internacionalidad de sus publicaciones. PUBLICATION QUALITY

The Editorial Universidad de Sevilla fulfils the criteria established by the National Commission for the Evaluation of Research Activity (CNEA) so that its publications are recognised as "of impact" (Ministry of Science and I nnovation, Resolution 18939 of 11 Novemb The Editorial Universidad de Serill operates a qualit control system which ensures the prestige and international nature of is publications, and is a member of the U.N. .E. (Unión de Editoriales Universitarias Españolas-Union of Spanish University Publishers.

Los contenidos de la revista PROYECTO, PROGRESO, ARQUITECTURA aparece en:

\section{bases de datos: indexación}

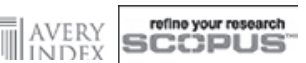 \\ iseceboaros [|[ e-revist@s \\ ProQuest' O Dialnet}

SCOPUS

AVERY. Avery Index to Architectural Periodicals

SSOC (Producida por el CCHS del CSIO)

-REVIST@S (CSIC)

DOAJ, Directory of Open Access Journals
PROQUEST (Arts \& Humanities, full text)

DRIJ. Directory of Research Journals Indexing

SJR (2014): $0.100, H$ index: 0

\section{catalogaciones: criterios de calidad}

RESH (Revistas Españolas de Ciencias Sociales y Humanidades).

Catálogos CNEAl (16 criterios de 19). ANECA (18 criterios de 21). LATINDEX (35 criterios sobre 36)

DICE (CCHS del CSIC, ANECA).

MIAR, Matriu d'I'Informació per a l'Avaluació de Revistes. IDCS 2015: 9,278. Campo ARQUITECTURA (internacional) 24/230

CIRUS, for Scientific Information

ULRICH'S WEB, Global Serials Directory

\section{catálogos on-line bibliotecas notables de arquitectura:}

CLIO. Catálogo on-line. Columbia University. New York

SBD Sistema Bibliotecario e Documentale Instituto Universitario di Architetura di Venezi

OPAC. Servizi Bibliotecari di Ateneo. Biblioteca Centrale. Politecnico di Milano

COPAC. Catálogo colectivo (Reino Unido)
SUDOC Catálogo colectivo (Francia)

ZBD Catálogo colectivo (Alemania)

REBIUN. Catálogo colectivo (España)

OCLC. WorldCat (Mundia)

\section{DECLARACIÓN ÉTICA SOBRE PUBUICACIÓN Y MALAS PRÁCTICAS}

(a) 作 Así nuestra revista garantiza lientificas define el COMITE DE EIICA DE PUBLICACIONES (COPE). publicado, protegiendo y respetando el contenido de los artículos y la integridad de los mismo. El Consejo Editorial se comEn

En cumplimiento de estas buenas prácticas, la revista PPA tiene publicado el sistema de arbitraje que sigue para la selección Consejo Editorial- La revista PPA mantiene actualizado estos criterios, basados exclusivamente en la relevancia cientifica de artículo, originalidad, claridad y pertinencia del trabajo presentado.

Nuestra revista garantiza en todo momento la condifencialidad del proceso de evaluación. el anonimato de los evaluadores y de los autores; el contenido evaluado; el informe razonado emitidos por los evaluadores y cualquier otra comunicación emitida

Igualmente queda afectado de la máxima confidencialidad las posibles aclaraciones, reclamaciones o quejas que un autor su contenido sea fraudulento, serán eliminados o no publicados de la revista PPA. La revista se identifiquen como plagio o mayor celeridad posible. Al aceptar los terminos y acuerdos expresados por nuestra revista, los autores han de garantizar

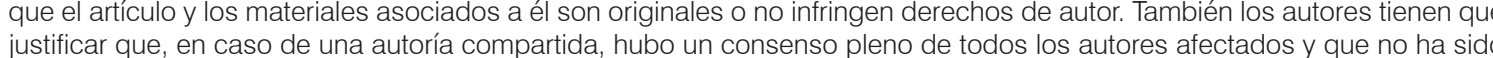
presentado ni publicado con anterioridad en otro medio de difusión.

ETHICS STATEMENT ON PUBLICATION AND BAD PRACTICES

PROYECTO, PROGRESO ARQUITECTURA (PPA) makes a commitment to the academic community by ensuring the ethics and quality of its published articles. As a benchmark, our journal uses the Code of Conduct and Good Practices which, for scientific journals, is defined for editors by the PUBLICATION ETHICS COMMITTEE (COPE). Our journal thereby guarantees an appropriate response to the needs of readers and authors, ensuring the quality of the pu-
blished work, protecting and respecting the content and integrity of the articles. The Editorial Board will publish corrections, clarifications, retractions and apologies when necessand

In compliance with these best practices, PPA has published the arbitration system that is followed for the selection of articles as solely on the scientific importance, the originality clarity and, relevance of the presented article-

Our journal guarantees the confidentiality of the evaluation process at all times: the anonymity of the reviewers and authors; the reviewed content; the reasoned
and scientific boards as required. Equally, the strictest confidentiality applies to possible clarifications, claims or complaints that an author may wish to refer to the

PROYECTO, PROGRESO ARQUITECTURA (PPA) declares its commitment to the respect and integrity of work already published will be eliminated or not published in PPA. The journal will act as quickly as possible in such cases. In accepting the terms and conditions expressed by our journal, authors must guarantee that the article and the materials associated with it are original and do not infringe copyright. The authors will also have to warrant that, in the case of joint authorship, there has been full consensus 
entre lineas LA ARQUITECTURA COMO MODO DE ENTENDER EL MUNDO. NOTAS DE UN PROFESOR
VETERANO / ARCHITECTURE AS A WAY TO UNDERSTAND THE WORLD. NOTES FROM A VETERAN

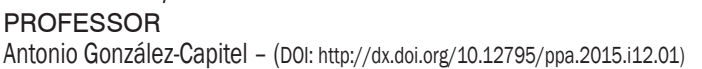

artículos
LA PALABRA DIBUJADA. ANTONIO FERNÁNDEZ-ALBA, PRIMER Y ÚLTIMO MAESTRO / THE SKETCHED WORD. ANTONIO FERNÁNDEZ-ALBA, THE FIRST AND LAST MASTER

CARVAJAL Y LA VOLUNTAD DE SER ARQUITECTO: LA CONSTRUCCIÓN DEL PROYECTO Y LA W AVAAL AND THE WILL TO BE ARCHITECT: THE CONSTRUCTION OF THE PROJECT AND EFFECTIVE BEAUTY
Carlos Labarta Aizpuń; Jorge Tárrago Mingo - (Dol: htpp://dx.doliorg 10.12795/ppa.2015.12.03) CIUDAD BLANCA EN BAHIIA DE ALCUDIA. UNA OBRA CON SENTIDO PEDAGOG ICO DEL
PROFESR FRANCISCO JAVIIER SÁENZ DE OIZZA. 1961 -63 / CIUDAD BLANCA IN ALCUDIA BAY, AN EDUCATIONAL WORK BY PROFESSOR FRANCIZCO JAVIER SAENZ DE OIZZA. 1961-63

SEVILLA Y EL SEVILLA 1(1972-2015)/ SEVILLE AND THE SEVILLE 1(1972-2015)

$$
\text { SEVILLA Y EL SEVILLA 1(1972-2015) / SEVILLE AND }
$$

DE LA PROFESIÓN A LA DOCENCIA: LOS VIAJES A INGLATERRA DE MANUEL TRILLO Y LAS VIVIENDAS EN LA MOTILLA / FROM PROFESSION TO TEACHING: MANUEL TRILLO AND HIS TRIPS TO

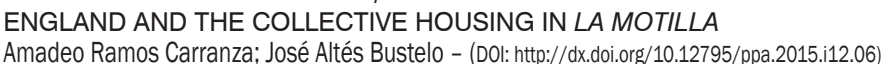

LA CONDICIÓN TERRITORIAL DE LO URBANO. EN TORNO A LA TRAYECTORIA DOCENTE DE PABLO ARIAS / THE CITY WITHIN THE FRAME OF TERRITORY. ABOUT THE ACADEMIC CAREER OF

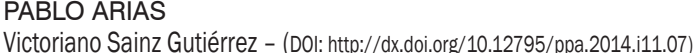

eña bibliográ́ica TEXTOS VIVOS

MANUEL TRILLO DE LEYVA: LA EXPOSICIÓN IBEROAMERICANA: LA TRANSFORMACIÓN

URBANA DE SEVILLA

MANUEL TRILLO DE LEYVA: CONSTRUYENDO LONDRES; DIBUJANDO EUROPA 


\section{LA ARQUITECTURA COMO MODO DE ENTENDER EL MUNDO.}

\section{NOTAS DE UN PROFESOR VETERANO}

ARCHITECTURE AS A WAY TO UNDERSTAND THE WORLD. NOTES FROM

A VETERAN PROFESSOR

Antón González-Capite

RESUMEN El autor sintetiza su posición y su historia acerca de la enseñanzza, la investigacion y el propio ejercicio de la arquitecura partiendo de los viejos tiempos de las primeras crisis modernas, donde el sentido de la arquitectura se había perdido casi, y como una disciplina de contenido propio, como un importante campo de conocimiento, ys de un privilesido modo de entender el mundo.

SUMMARY The author summarizes his position and his story about teaching, research and the actual practice of architecture. Starting from the old days of the first modern crisis, where the sense of architecture was almost lost, and its recovery, through the KEY WORDS Architect

Persona de contacto/Corresponding autor: antoncapitel@gmail.com. Escuela Técnica Superior de Arquitectura. Universidad Politécnica de Madrid.
E $\mathrm{n}$ 1971, la Escuela de Arquitectura de Madrid estaba vacía de ideología; o, al menos, de ideologia que fuera sallstactoria. Los tardios años sesenta la hablan convanido en un desierto. Por un lado aquellas cosas de Archigram, y tantas, y tantas otras vanguardias, por otro, la semilologia, la sociologia, la polica, Alexander, los ordenadores, el neo-funcionalismo, e estructuralismo... iu!! Tantas, demasiadas cosas. Tantas que desaparecieron; un viento, quizá ligero, se las llevó sin esfuerzo. Eran bienes volanderos. En 1971 no quedaba nada.

Pero la arquitectura misma, la arquitectura buena, convencional o no, tambien se había ido. El movimiento modeno, después del organilismo, habla llegado a una situación muy eclectica y había dosaparecido. Al menos hubla desaparecido en las concienclas de aquellos que, may jovenes, empezaban os entonces a ocupar las Esculas. Nos lataron, les haciamos caso y las ocupábacer iDónd está a arquitectura? iEn quéconsiste? cer. ¿Dón úlo éála arquitectra? ¿En qué consiste?

de prestigio internalo - de prestig intes acional reciente -Siling- ocupaban Aalto, Utzon Kahn .... Pero pronto, nos dimos cuenta

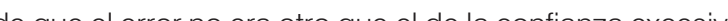

y exclusiva en lo contemporáneo, en lo último, en lo más original y novedoso, que ponia en duda todo lo anterior Pus la arquieclua estaba, en realidad, en muchos sitios: en las ciudades y en los libros, por ejemplo, y sobre todo. Fuimos de viaje, paseamos calles y entramos en librelas, y empezamos a ver que, para nuestra tranquilidad, la arquilectura estaba alli. No era un misterio, podía verse y hasta tocarse, disculir sobre ella, conocerse, analizarse. Analizar, mostrar, explicar la arquitectura buena de los dilatados y diversos tiempos modernos se convirtio pronto en nuestro recurso principal. No importaba e tiempo en que la arquilectura se hubiera producido, no importaba su tendencia, o su clase, solo importaba que Y

Yaparecieron, por fortuna, nuevos profetas, capaces de consoldar algo aquellas inicicones. Uno nacional afre pán y que explicha y nos ayna na pero que no lo era. ces considerados profetas mayores a los extranieros

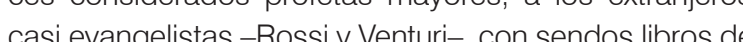
mismo ano, 1906, peri que pul, con sendos libros del sól en los primeros años 70 . Rossi y Venturi parecín

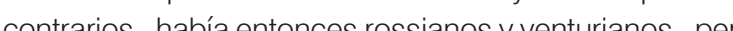
no 10 eran, tean complems 
arquitectura -y la ciudad- como tal, como una cuestión de forma. De forma no banal, de forma profunda, con contenido propio, y como principio de la arquitectura mis

Por que vís cencillos, pero inces "disciplina".

Por estas Vlas, sencllas, peri a ansas y eficaces, las Escuelas -la Escuela de Madrid, al menos- fueron reciench propio, pero que tan degen a propio, per quélo docos años a las Los nuevos profesocos aliendo adelante y fueron también creciendo. El allivo del conocimiento arqutectónico, incubado en las Es-

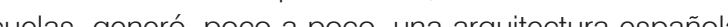
ens, enovada a do este feńmo, ah fo tiene una crisis ectéctica hab́ dejaco el panorama huérfano, vać Pero la lucidezla que la arquitectura era como tení que ser distinta, rezoada, variada pero como siempre al fin y al cabo, llenó cte vaćo con el imprescindible apoyo del conocimiento la reflexión y el trabajo. Y estas cosas, con toda lónica, hicieron milagros. Milagros ya bien conocidos.

Y esas cosas nos sostienen todaví podrí decirse Saber que nos interesa toda la arquitectura toda la de todos los tiempos. Que nos interesa conocerla examinara, analizarla, aprender de ella, conocer sus instrumentos, sus métodos, sus procedimientos, sus recursos, sus hallazgos, La de todos los tiempos, pues la naturaleza de la arquitectura y sus enseñanzas no cambia tanto como parecería que lo hace al contemplar circunstancias, modos de vivir materiales, estilos, ideales, tan, tan distintos como se van produciendo a lo largo del tiempo. Que la historia de la arquitectura probablemente no sivva para nada práctico, que no sivve, desde luego, para proyectar, pero que resulta imprescindible para entender disciplina misma, para conocer el mundo.

Saber que la sabiduría implícita en la arquitectura es real, que contiene conocimientos y contenidos reales. Que la arquitectura avanza siempre, pues es empírica, se produce por la práctica, pero que se ilumina mediante la teoría y la crítica que esa práctica suscitan. Que no se puede hacer un auditorio, por ejemplo, ignorando los hallazgos de Aalto o de Scharoun, como vemos en algunos sedicentes contemporáneos. Que solo puede ignorarse aquello que realmente se co-

Saber, pues, que la arquitectura es conocimiento y trabajo, más que habilidad innata, condición artística, novación no puede ser un. Que en la arquilectura la inque tanno puede cín puede ser un fin pues debe de tratarse tan ślo de un acontecimiento. Que sól la más alla calidad posible es el fin único y acertado ¿Que dús es calidad? dicen usedes. IAy anigos! A eso sólo se es callad? dicen ustedes. iAy, amigos! A eso sólo se

tro pro ectura como la angustioso transourso por al canzar dominio de tipos y de procedimientos, como la historia de los fracasos y como la historia do haber ido logrondo paulatinamente, el dominio de antiguas ambiciones ar quitectónicas, antes muy torpemente conocidas. El siglo XXI es fecundo pero no tanto por las ansiosas muestras de originalidad -aunque algunas estimables y atractivas otras muchas ridículas, superfluas y nocivas-, sino por ser el heredero de una historia de la arquitectura tan rica Sobre todo por ser el heredero del siglo XX en el que a revolución arquitectónica moderna produjo la mayor cantidad, calidad y diversidad de arquitecturas cualificadas de ningún otra época en la historia. Quien ignore las radiciones modernas del siglo XX ignorará el contenido mismo de su propio quehacer, ignorará la arquitectura, no entenderá el mundo

Así, pues, conocimiento. Profundizar en la arquitectura no tanto, o no sólo, como una actividad profesional técnica y artística, si no como una cultura, como un campo de conocimiento, como un modo, también, de entender el mundo.

La arquitectura como estudio, como acumulación de sistemas, como teoría. Dicen que no hay teoría, tantos lo dicen, convencidos de que el transcurso del tiempo todo lo supera, todo lo deja atrás. Puede que así sea, pero no es por esto por lo que no hay teoría, no la hay porque nadie, casi nadie, se dedica a ella. $O$, si lo hace, la teoría se entiende como una reflexión especulativa, apoyada en la osofla, y muy lejana, lejanisima, de la arquitectura real,
Es necesario defender la teoría como la palabra que a arquitectura no tiene. Las arquitecturas que nos interean son inmano liene. Las arquitecturas que nos interecomplementars, Las teoŕas deberín serlo Las teo-

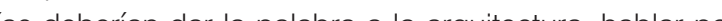
ella, explicarla desentrañar sus métodos y sus logros, re lexionar de forma concreta sobre lo que han alcanzado. Defendemos la teoŕa como algo parecido a lo que los "ilianos de Ios años 60 llamaban "teoŕa del proyecto", 0 "crítica operativa", que Tafuri, con su artillería ideológica 'ridiculizó y pulverizó para introducir un modo de ver lejno, lejańsimo, de entenderla desde presupuestos ideológicos y filosóficos que acaso ayuden a deteminada concepciones del mundo, pero no tanto, casinada a a interés.

"Sublime inutilidad" llegó a decir Tafuri de la arquitecdel revés ese agresivo ataque y sin que nos mueva la crueldad, dedicar su epíteto a sus propios ensayos: sobre todo, desde luego a aquellos que pretendían ser más profundos, y que fueron en buena medida responsables del modo en que la teoría la inńtil teoría, se ha extendido desde el mundo anglosajón. Hoy las facultades y escue las de arquitectura, ebrias de teorías filosóficas, buscan inútilmente lo que podrían tener en realidad, entre sus manos, si no hubieran sido como las vírgenes necias. En los años 70, los rossianos hablaban de una "refundación disciplinar", en buena medida aplicada en su momento, y como ya se ha dicho o insinuado. Pero, después de tantos años, la creencia en que el campo de la arquitectura sea algo propio y consistente, se ha quebrado, al menos para determinadas generaciones. Acaso la escasa importancia que parece tener la buena arquitectura para la sociedad que ha de hacerla posible haya influido en esta falta de fe. Y como la posible bondad de nuestras sociedades y de sus políticas no parece estar en buenos momentos, acaso hayamos de esperar lo peor: la desaparición paulatina, en buena medida consumada del campo arquitectónico entendido como una cultura Pero, si es así, más nos compete todavía guardar, como si fuéramos frailes medievales, el tesoro del conocimiento que somos capaces de vislumbrar. Se impone, pues, una nueva refundacion, o restauracion de la disciplina. Ahora sería más fructifera, porque tantas cosas intensas e imtantes se han hecho en estas décadas.

A veces siento pena por los estudiantes de ahora, oficio que sigo ensenando con el mismo entusiasmo el probablemente la mayor parte no tengan oportunich de ejrcerlo. Pero tambín he enseñado, en todos estos años, a entender el munclo a través de la arquitectura taly como yo mismo he hecho y gozado: o dicho de otro modo a entender que ba a vurectura trasciende ese ficio, no tanto porque tenga muchísima importancia sino porque tiene una gran profundidad oran amplitud y atractivo especial por sus valores genuinos En símisma en su propia materialidad y precisión, en su concreción. La arquitectura es una convención humana una obra humana tan artificiosa $y$ tan voluntaria como la gastronomía, pongo por caso no tan leiano Y del mismo modo que sin convertir en arte el hecho de comer se puede vivi bien (véase, si no a británicos, estadounidenses, holandeses, -) también sin buena arquitectura -la conversión de la necesidad del cobijo en un arte- se podría vivir. Por eso está, y estará siempre, en peligro.

Entender el mundo a través de la arquitectura, hacer que la arquitectura explique el mundo. He intentado ha cerlo estudiando la arquitectura española, tanto en modo general como en términos monográficos, para intenta explicar el mundo producido por mis mayores y por mis contemporáneos. He intentado hacerlo de un modo crítco, pues es éste, creo, el único que alcanza el verdadero conocimiento.

He trabajado también sobre la arquitectura moderna universal, sobre algunos de aquéllos a los que tanto admiramos. Quisiera tener más vidas sólo para seguir haciéndolo.

He creído descubrir campos en los que la arquitecura se ha manifestado con amplio alcance, tal y como cuando ha tenido que intervenir para transformar positvamente cualificadas obras del pasado; o cuando se ha inspirado en formas ilusorias y figuras de lenguaje para acercarse a mundos como lo misterioso y lo poético y iterario que parecían fuera de su alcance. He razonado so la condición impura de la disciplina arquitectónica

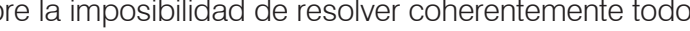
sus dispares requisitos, y, así, sobre aquellos métodos 
ideas con los que los arquitectos han salido al paso de ste problema.

He pretendido clasificar y analizar algunos de sus métodos, estudiando el ancestral arquetipo de sus palios, de la más remola aniguedad hasta to conpartes, casi tan universal; o el ideal de la arquitectucompacta igulmente extendido por arquitecturas muy diversas. Sé que no todo se puede sistemastizas acaso ni siquiera esto que he dicho, y que he hecho. Pero tambín sé que al orden, el méloco y una atención indiscriminada al pasado a la moderidad y a presente resultan enriquecedores para nuestros fines (n)
He escrito casi sin descanso desde hace muchísimos años. En los papeles está, $y$, como a Machado, me debéis lo que he hecho. Pero no pretendo otro pago que el de lo fructifero de mi ejemplo: que crean las generaciones posteriores en que la arquitectura, tal y como se le lo cotidiano a lo sublime merece la pena. No sól de

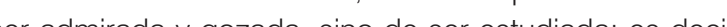
entendida y explicada, para el progreso acunulativo nestro campo. Para llustracín pejenp ac de escéplicos, expertos y profanos.

- Ahora que todo lo profesional pierde fuerza, ahora (a)

Antonio González-Capitel (1947), arquitecto y profesor de la Escuela Tecnica Superior de Arquitectura de Madrid. Profesor invtado y visitante en numeriosas universidades espanolas, europeas y americanas. Arquitecto en ejercicicio. Investigador con numerosos escritosy ilibros sobre arquitectura modema espanola einternacional. Ha sido hspector General de Monumentios del Estado (España) director de la revista "Arquitectura" del coles 


\section{LA ARQUITECTURA COMO MODO DE ENTENDER EL MUNDO.}

CRCHTECTURE AS A WAY TOUNDEST

NOTES FROM A VETERAN PROFESSOR

NOtón González-Capitel

p.19 In 1971 , the School of Architecture of Madrid lacked ideology; or, at least, an ideology that was satisfactory. The late sixties hac theded it into a desert. On the one hand those things of Archigram, and many, many others, avant-garde; secondly, semiotics

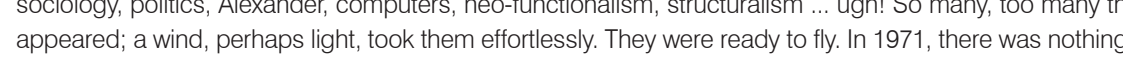

Architecture itself, good architecture, conventional or not, was gone too. The modern movement, after organicism, had reached a very eclectic situation and had gone. At least it had disappeared in the consciences of those who, when very young One is architecture? What does it consist

作 exclusive reliance on the contemporary, in the latest, in the most original and new calling into gone other than the excessive and before. Architecture was, in fact, in many places: in cilies and above all in books. We travelled, we wandered streets and into bookstores, and begin to see that, for our peace of mind, architecture was there. It was not a mystery, it could be seen and even 政 class, it only mattered that it seemed interesting, qualified, attractive.

Fortunately, new prophets appeared that were able to consolidate something from those intutitions. One of those was a national, who lived hallway between Madrid and Barcelona - Moneo-, and who then seemed minor, but was not. He explained and

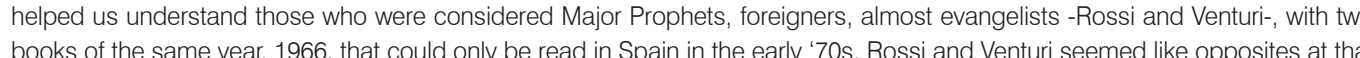
books of the same year, 1966, that could only be read in Spain in the early 70 s. Rossi and Ventur seemed like opposites at that

p.20 defended the architecture -and the city-as such, as a matter of form. Not in a trivial way but deeply, with its own contents and as

a beginning of architecture itself, what then began to be referred to as "discipline".
In these simple but ingenious and effective ways, the schools- The School of Madrid, at least, were receiving new and solid substance and began to grow in the knowledge of a field that was ultimately its own, but that was so degenerate or so evasive only
a few years earier. The new professionals-and some that already were, although few-were moving forward and were growing. The cultivation of architectural knowledge incubated in schools, gradually generated a renewed Spanish architecture that before long received international recognition. For those who do not know how this phenomenon was created, there you have it: an eclectic crisis had left an orphan, emply landscape. However, the lucidity of that architecture was as it should have been different, renewed, varied, but as always, at the end of the day, filling this void with the essential support of knowledge, reflection and work. These

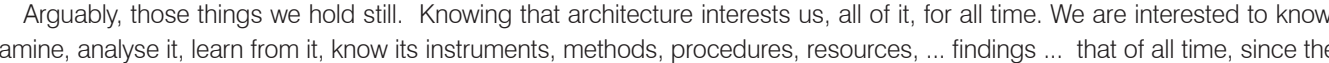
nature of architecture and its teachings does not change as much as contemplating the circumstances, lifestyles, materials, styles and ideals does; so, so different as they are created over time. The history of architecture perhaps has no practical purpose,

Knowing that the implicit knowledge in architecture is real, it contains knowledge and real content. Architecture is always cannot make an auditorium, for example, ignoring the findings of Aatto or Scharoun, as we see with some would-be contemporaries. That can only ignore what is really known.

ge and work, rather than an innate ability, artistic condition, originality or innovation. In archality is possible and the only successful end. What is cur cality? creation be an end, it must simply be an event. Only the highes with everything, with knowledge.

Is there progress? Of course, there is, and it cannot be otherwise. The history of architecture could be written like the anguished process th achieving mastery of ypes and procedures, ike the history of falures and like the history of having gradually achieved the mastering of ancient architectural ambitions that in the past were very awkwardly known. The twentieth century is
fertile, but not for the anxious samples of originality- though some worthy and attractive, many others ridiculous, unnecessary and damaging-but for being the heir of such a rich architectural history. Above all, as the heir of the twentieth century in which the modern architectural revolution produced the greatest quantity, quality and diversity of qualified architectures at no other time in history. Those who ignore modern traditions of the twentieth century will ignore the very content of one's own work, ignore archtecture and will not understand the world
Therefore, knowledge To delve into cuture, as a field of knowledge, as a way to also understand the world

with the passing of time everything exceeds, everything is left behind. It may be so, but this is not the reason why there is no theor it is because no one, almost no one, is dedicated to it. If they do, the theory is understood as a speculative reflection, based on Ature as such.

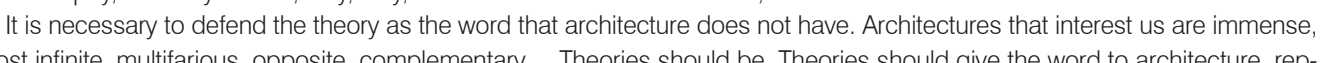
resent it, explain it, unravel its methods and achievements and specifically reflect on what it has achieved. We defend the theory similar to what the Italians in the 60s called "theory of project" or "operational critifue", which Tafuri, with his ideological artillery,

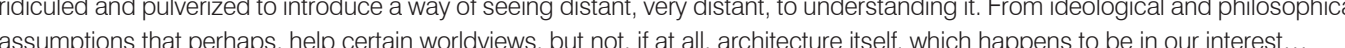

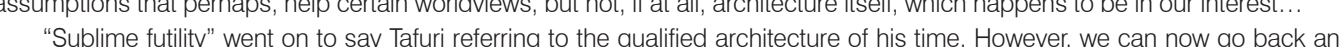
reverse this aggressive attack and without being cruel, dedicate his epithet to his own experiments; especially, of course, the ones that claim to be deeper, and were largely responsible for the way in which theory, useless theory has spread from the Anglo world. Today the faculties and schools of architecture, inebrialed from philosophical theories, seeking in vain what they could have actually had in their hands, if they were not like the foolish virgins.

"istablishing discipline" largely applied at the time, and as aready stated or implied. However, after so many years, the belief in that the field of architecture is something proper and consistent makes it possible has contributed to this lack of fatth. As the possible kindness of our societies and their policies currently do not appear to be at their best, perhaps we have to expect the worst: the gradual disappearance, largely consumed, the architectural
field understood as a culture. However, if this is the case, even more so we must keep, as if we were medieval monks the treasure of knowledge that we are able to glimpse A new re establishing or restoration of discipline is therefore essential Now, the treasure more fruitty because so many intense And mportant things have been done in recent decades Sometimes I feel sorry for the students now, which I still teach with the same enthusiasm the profession that I believe in, but aware that probably most will not have the opportunity to practice it. However, I have also taught, over the years, to understand

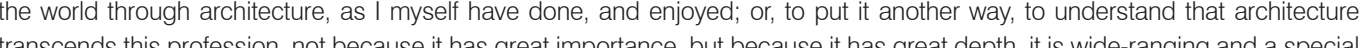
transcends this profession, not because it has great importance, but because it has great depth, it is wide-ranging and a special
appeal for their true values. In itself, in its own materiality and accuracy, in its realisation. The architecture is a human convention, a human work, so contrved and as voluntany as tood put by case, not so far In the same way and without turning eating into an art you can live well (see, if not, the British, American, Dutch...) also without good architecture -the conversion of the need for shelter

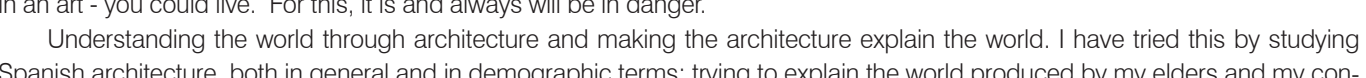
Spanish architecture, both in genetral and in demographic terms; trying to explain the world produced by my elders and my con-

Ihave also
continue to doit.

7 thought to find areas in which the architecture is manifested with ample reach, as when it had to intervene to transform positively skilled works of the past; or when it was inspired by illusory forms and figures of speech to approach mysterious, poetic
and literan worlds that seemed out of reach, I have reasoned on the impure condition of the architectural discipline inability to consistently solve all its absurd requirements, and so, on those methods and ideas with which architects have come out against p.22 this problem

I have tried to classify and analyse some of their methods, studying the ancient archetype of their courtyards, from the re-

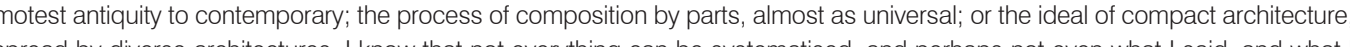
have done. However I also know that the order, method and indiscriminate attention to the past. to modernity and the present are of knowledge.

I have written almost nonstop for many years. On paper, it is, and like Machado, you owe me for what I have done. However, Id not expect another payment ther than the fruituluness of my example. creating subsequent generations where architecture, as occurs in many fortunate and such difiterent cases, from the everyday to the sublime, is worth it. Not only to be admired and non-professionals.

Now that all things prolessional are losing strength, now that we do not build as much, as it seems, it could be the great opportunity of reflection and study. 